Acta Crystallographica Section B

Structural

Science

ISSN 0108-7681

Editor: Carolyn P. Brock

\title{
[18]-Annulene, $\mathrm{C}_{18} \mathrm{H}_{18}$, structure, disorder and Hückel's $\mathbf{4 n}+\mathbf{2}$ rule
}

S. Gorter, E. Rutten-Keulemans, M. Krever, C. Romers and D. W. J. Cruickshank

This electronic document was scanned from an archival copy of material deposited to accompany a paper published in an IUCr journal. In many cases the only accessible copy was a microfilm of a poor-quality original. 


\section{Tables for deposinion}

Table 5.

Intramolecular distances for major and minor molecules in A. C.H distances in the minor molecule have been fixed at 1.00 A. No ess values are displayed for the minor molecule distances.

$\begin{array}{lll}C(1) & -C(2) & 1.390(3) \\ C(1) & -H(1) & 0.99(1) \\ C(1) & -C\left(9^{1}\right) & 1.382(1) \\ C(2) & -C(3) & 1.401(2) \\ C(2) & -H(2) & 0.99(2) \\ C(3) & -C(4) & 1.386(2) \\ C(3) & -H(3) & 0.95(2) \\ C(4) & -C(5) & 1.386(2) \\ C(4) & -H(4) & 0.99(2) \\ C(5) & -C(6) & 1.403(2) \\ C(5) & -H(5) & 0.98(1) \\ C(6) & -C(7) & 1.387(2) \\ C(6) & -H(6) & 1.00(2) \\ C(7) & -C(8) & 1.377(2) \\ C(7) & -H(7) & 0.97(2)\end{array}$

$\begin{array}{lll}C(8)-C(9) & & 1.411(2) \\ C\left(8^{\prime}\right)-H(8) & & 0.97(2) \\ C(9)-1(9) & & 0.96(2) \\ C(9)-C\left(1^{\prime \prime}\right) & & 1.382(2) \\ C\left(1^{\prime}\right)-C\left(2^{\prime}\right) & & \\ C\left(1^{\prime}\right)-C\left(9^{\prime \prime}\right) & & 1.391 \\ C\left(2^{\prime}\right)-C\left(3^{\prime}\right) & & 1.377 \\ C\left(3^{\prime}\right)-C\left(4^{\prime}\right) & & 1.377 \\ C\left(4^{\prime}\right)-C\left(5^{\prime}\right) & & 1.384 \\ C\left(5^{\prime}\right)-C\left(6^{\prime}\right) & & 1.380 \\ C\left(6^{\prime}\right)-C\left(7^{\prime}\right) & & 1.385 \\ C\left(7^{\prime}\right)-C\left(8^{\prime}\right) & \ddots & 1.378 \\ C\left(8^{\prime}\right)-C\left(9^{\prime}\right) & : & 1.399 \\ C\left(9^{\prime}\right)-C\left(1^{\prime \prime}\right) & & 1.377\end{array}$

\section{Table 6.}

Intramolecular bond angles in degrees.

\begin{tabular}{|c|c|c|c|c|c|c|c|}
\hline$C(2)$ & $C(1)$ & $-H(1)$ & $114.9(8)$ & $C\left(2^{\prime}\right)$ & $C\left(1^{\prime}\right)$ & $\mathrm{H}\left(1^{\prime}\right)$ & 115.6 \\
\hline$C(2)$ & $-C(1)$ & - $C\left(9^{\prime \prime}\right)$ & $127.1(1)$ & $C\left(2^{\prime}\right)$ & $-C\left(1^{\prime}\right)$ & $C\left(9^{\prime \prime \prime}\right)$ & 128.9 \\
\hline $\begin{array}{l}\mathrm{H}(1) \\
\mathrm{C}(1)\end{array}$ & $-C(1)$ & $-C\left(9^{\prime \prime}\right)$ & $118.1(8)$ & $\mathrm{H}\left(\mathrm{I}^{\prime}\right)$ & $-C\left(1^{\prime}\right)-$ & $\mathrm{C}\left(9^{\prime \prime \prime}\right)$ & $\begin{array}{l}115.3 \\
1183\end{array}$ \\
\hline $\begin{array}{l}\mathrm{C}(1) \\
\mathrm{C}(1)\end{array}$ & $-C(2)$ & $-C(3)$ & $\begin{array}{l}124.1(1) \\
1183199\end{array}$ & $\begin{array}{l}H\left(2^{\prime}\right) \\
H\left(2^{\prime}\right)\end{array}$ & $\begin{array}{l}=C(2) \\
-C(2)\end{array}$ & $\begin{array}{l}C(1) \\
C\left(3^{\prime}\right)\end{array}$ & $\begin{array}{l}118.3 \\
115.7\end{array}$ \\
\hline $\begin{array}{l}C(1) \\
C(3)\end{array}$ & $\begin{array}{l}-C(2) \\
-C(2)\end{array}$ & $\begin{array}{l}-H(2) \\
-\quad H(2)\end{array}$ & $118.8(9)$ & $C\left(1^{\prime}\right)$ & $-C\left(2^{\prime}\right)$ & $\mathrm{C}\left(3^{\prime}\right)$ & \\
\hline $\mathrm{Cl}$ & $-C(3)$ & $-C(4)$ & $124.0(1)$ & $\mathrm{H}\left(3^{\prime}\right)$ & $-C\left(3^{\prime}\right)$ & $C\left(2^{\prime}\right)$ & \\
\hline $\mathrm{C}(2)$ & $-c(3)$ & $-H(3)$ & $117.4(9)$ & $H(3)$ & $-C\left(3^{\prime}\right)$ & $C\left(4^{\prime}\right)$ & \\
\hline$C(4)$ & $-C(3)$ & $-H(3)$ & $118.6(9)$ & $C\left(2^{\prime}\right)$ & $-C\left(3^{\prime}\right)$ & C(4) & 8 \\
\hline$C(3)$ & $-C(4)$ & $-C(5)$ & $128.5(1)$ & $C\left(3^{\prime}\right)$ & $-\quad C\left(4^{\prime}\right)$ & & \\
\hline$C(3)$ & $-C(4)$ & - $\quad \mathrm{H}(4)$ & $116(1)$ & $\begin{array}{l}C\left(3^{\prime}\right) \\
C\left(5^{\prime}\right)\end{array}$ & $\begin{array}{l}-C(4) \\
-\quad C(4)\end{array}$ & $\begin{array}{l}H(4) \\
H(4)\end{array}$ & $\begin{array}{l}11.48 \\
115.8\end{array}$ \\
\hline$C l$ & $\begin{array}{l}-C(4) \\
-\quad C(5)\end{array}$ & $\begin{array}{l}-H(4) \\
-C(6)\end{array}$ & $\begin{array}{l}15(1) \\
123.4(1)\end{array}$ & $\mathrm{H}\left(5^{\prime}\right)$ & $-C\left(5^{\prime}\right)$ & $C\left(4^{\prime}\right):$ & 8 \\
\hline$C+1$ & $-C(5)$ & $-H(5)$ & $117(1)$ & $\mathrm{H}\left(5^{\prime}\right)$ & $-C\left(5^{\prime}\right)$ & $C\left(6^{\prime}\right)$. & .0 \\
\hline$C(6)$ & $-C(5)$ & $-H(5)$ & $119.1(9)$ & $C\left(4^{\prime}\right)$ & $-C\left(5^{\prime}\right)$ & $C\left(6^{\prime}\right)$ & \\
\hline$C(5)$ & $-c(6)$ & $-C(7)$ & $124.1(1)$ & $H\left(6^{\prime}\right)$ & $-C\left(6^{\prime}\right)$ & $C(5)$ & \\
\hline$(15)$ & $-C(6)$ & $-\mathrm{H}(6)$ & $119.5(9)$ & $\begin{array}{l}I I\left(6^{\prime}\right) \\
C\left(5^{\prime}\right)\end{array}$ & $\begin{array}{l}-C(0) \\
-C(6)\end{array}$ & (4) & 124.9 \\
\hline$C(7)$ & $\begin{array}{l}-C(6) \\
-C(7)\end{array}$ & $\begin{array}{l}=1(6) \\
-\quad C(8)\end{array}$ & $\begin{array}{l}116.4(9) \\
128.2(1)\end{array}$ & $C(6)$ & $-C\left(7^{\prime}\right)$ & $C(8)$ & 128.0 \\
\hline Co & $=0(7)$ & $-1(7)$ & $115.5(9)$ & $C\left(6^{\circ}\right)$ & $-C\left(7^{\prime}\right)$ & $\mathrm{H}(7)$ & 4 \\
\hline$C(8$ & $=C(7)$ & $-\mathrm{H}(7)$ & $116.2(9)$ & $C\left(8^{\circ}\right)$ & $-C\left(7^{\prime}\right)$ & $\mathrm{H}(7)$ & 6.6 \\
\hline $\mathrm{Cl}$ & $-c(8)$ & $-C(9)$ & $124.0(1)$ & $\mathrm{H}(8)$ & $-C(8)$ & $\begin{array}{c}C(7) \\
C(9)\end{array}$ & 7.8 \\
\hline $\mathrm{Cl}$ & $-C(8)$ & $-H(8)$ & $116.7(9)$ & $\begin{array}{l}17(8) \\
672\end{array}$ & $\begin{array}{l}-48 \\
-9(8)\end{array}$ & C. & 4.3 \\
\hline 0 & - C(8) & $\begin{array}{l}-H(8) \\
-H(0)\end{array}$ & $\begin{array}{l}19.5(9) \\
116809\end{array}$ & $C\left(8^{\prime}\right)$ & - C(9' & $C\left(1^{\prime \prime \prime}\right)$ & 125.3 \\
\hline S & $\begin{array}{l}-C(9) \\
-\quad(9)\end{array}$ & $-C\left(I^{\prime \prime}\right)$ & $124.7(1)$ & $C\left(8^{\circ}\right)$ & $-C\left(9^{\prime}\right)$ & $\mathrm{H}\left(9^{\prime}\right)$ & \\
\hline 40 & $C(9)$ & - C(1") & $118.4(9)$ & (1'i') & $C\left(9^{\circ}\right)$ & $H\left(9^{\prime}\right)$ & 18 \\
\hline
\end{tabular}

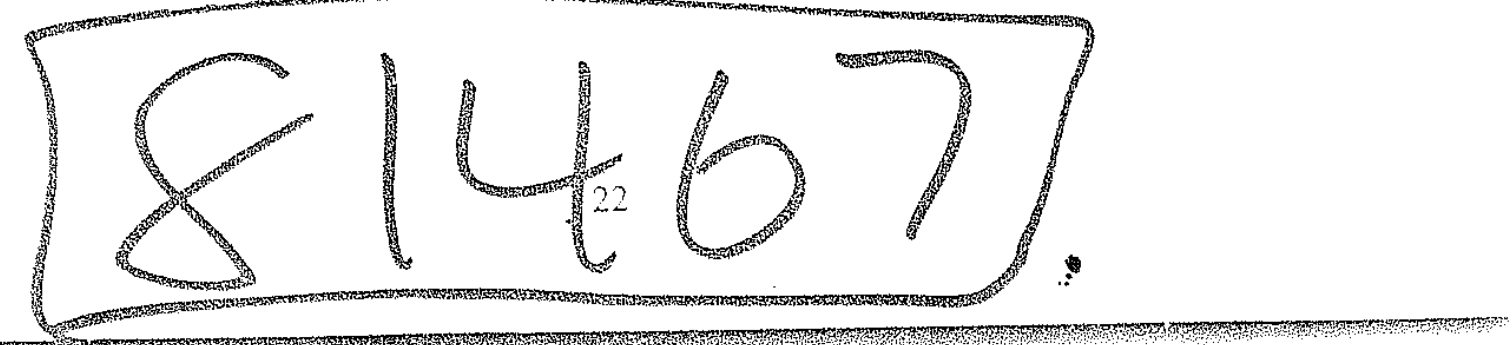




\section{Tabie 7}

Thermal parameters for mojor and minor molecule of $\mid 18$-ammulene.

All thermatl parameters $\times 10^{4}$.

\begin{tabular}{|c|c|c|c|c|c|c|}
\hline ATOM & $11(11)$ & U(22) & $0(33)$ & $2(121)$ & $2 U(23)$ & $211(31)$ \\
\hline $\begin{array}{l}C(1) \\
C(2) \\
C(3) \\
C(4) \\
C(5) \\
C(6) \\
C(7) \\
C(8) \\
C(9) \\
C\left(1^{\prime}\right) \\
C\left(2^{\prime}\right) \\
C\left(3^{\prime}\right) \\
C\left(4^{\prime}\right) \\
C\left(5^{\prime}\right) \\
C\left(6^{\prime}\right) \\
C\left(7^{\prime}\right) \\
C\left(8^{\prime}\right) \\
C\left(9^{\prime}\right)\end{array}$ & $\begin{array}{l}204(6) \\
23.160 \\
393(7) \\
334(6) \\
345(6) \\
285(6) \\
271(5) \\
258(5) \\
267(6) \\
328 \\
828 \\
337 \\
327 \\
201 \\
300 \\
300 \\
536 \\
319\end{array}$ & $\begin{array}{l}294(6) \\
351(7) \\
323(6) \\
362(7) \\
438(7) \\
406(8) \\
347(7) \\
334(6) \\
315(6) \\
345 \\
87 \\
492 \\
303 \\
757 \\
187 \\
302 \\
367 \\
450\end{array}$ & $\begin{array}{l}377(7) \\
343(7) \\
317(6) \\
237(5) \\
224(5) \\
261(6) \\
280(5) \\
388(6) \\
498(8) \\
551 \\
567 \\
453 \\
391 \\
526 \\
560 . \\
385 \cdot \\
934 \\
309\end{array}$ & $\begin{array}{c}-20(10) \\
129(11) \\
214(11) \\
189(11) \\
254(13) \\
167(11) \\
46(11) \\
2(11) \\
-95(11) \\
86 \\
102 \\
254 \\
140 \\
206 \\
140 \\
4 \\
305 \\
159\end{array}$ & $\begin{array}{c}35(11) \\
261(11) \\
220(10) \\
101(10) \\
75(11) \\
-154(11) \\
-19(11) \\
-195(12) \\
-90(12) \\
79 \\
-6 \\
-3 \\
16 \\
-583 \\
1 \\
-137 \\
370 \\
298\end{array}$ & $\begin{array}{c}206(10) \\
305(11) \\
353(11) \\
218(9) \\
154(10) \\
35(9) \\
115(9) \\
127(10) \\
330(12) \\
488 \\
901 \\
334 \\
178 \\
62 \\
97 \\
271 \\
.1087 \\
322\end{array}$ \\
\hline
\end{tabular}




\section{Table 8: List of structure factors 2 10 of [18]-Anwulene}

$\begin{array}{rrrrrr}H & K & 1 & 1 & & \\ 2 & 0 & 0 & 217 & 210 & a_{w} \\ 4 & 0 & 0 & 514 & 486 & 18 \\ 6 & 0 & 0 & 174 & 166 & 6 \\ 8 & 0 & 0 & 20 & 20 & 1 \\ 10 & 0 & 0 & 40 & 39 & 2 \\ 14 & 0 & 0 & 54 & 53 & 2 \\ 16 & 0 & 0 & 22 & 23 & 2 \\ 18 & 0 & 0 & 22 & 22 & 3 \\ 1 & 1 & 0 & 273 & 257 & 10 \\ 2 & 1 & 0 & 368 & 351 & 13 \\ 3 & 1 & 0 & 503 & 476 & 18 \\ 4 & 1 & 0 & 48 & 50 & 2 \\ 5 & 1 & 0 & 79 & 80 & 3 \\ 6 & 1 & 0 & 149 & 139 & 5 \\ 7 & 1 & 0 & 17 & 16 & 1 \\ 8 & 1 & 0 & 92 & 92 & 3 \\ 9 & 1 & 0 & 28 & 26 & 1 \\ 10 & 1 & 0 & 53 & 52 & 2 \\ 12 & 1 & 0 & 101 & 103 & 4 \\ 13 & 1 & 0 & 158 & 160 & 6 \\ 14 & 1 & 0 & 42 & 42 & 2 \\ 15 & 1 & 0 & 27 & 27 & 2 \\ 16 & 1 & 0 & 19 & 21 & 2 \\ 17 & 1 & 0 & 82 & 81 & 3 \\ 18 & 1 & 0 & 51 & 48 & 2 \\ 0 & 2 & 0 & 217 & 219 & 8 \\ 1 & 2 & 0 & 22 & 27 & 1 \\ 2 & 2 & 0 & 21 & 14 & 1 \\ 3 & 2 & 0 & 191 & 186 & 7 \\ 4 & 2 & 0 & 123 & 119 & 4 \\ 5 & 2 & 0 & 205 & 200 & 7 \\ 6 & 2 & 0 & 165 & 160 & 6 \\ 7 & 2 & 0 & 63 & 60 & 2 \\ 8 & 2 & 0 & 100 & 97 & 4 \\ 9 & 2 & 0 & 77 & 76 & 3 \\ 10 & 2 & 0 & 185 & 191 & 6 \\ 11 & 2 & 0 & 52 & 51 & 2 \\ 12 & 2 & 0 & 21 & 22 & 2 \\ 13 & 2 & 0 & 19 & 21 & 2 \\ 14 & 2 & 0 & 119 & 122 & 4 \\ 16 & 2 & 0 & 34 & 34 & 2 \\ 15 & 2 & 0 & 21 & 26 & 3 \\ 1 & 3 & 0 & 43 & 43 & 2 \\ 2 & 3 & 0 & 105 & 102 & 4 \\ 3 & 3 & 0 & 91 & 90 & 3 \\ 4 & 3 & 0 & 113 & 110 & 4 \\ 5 & 3 & 0 & 32 & 31 & 1 \\ 7 & 3 & 0 & 78 & 78 & 3 \\ 8 & 3 & 0 & 89 & 88 & 3 \\ 9 & 3 & 0 & 99 & 102 & 3 \\ 10 & 3 & 0 & 30 & 31 & 1 \\ 11 & 3 & 0 & 50 & 49 & 2 \\ 13 & 3 & 0 & 63 & 63 & 2 \\ 14 & 3 & 0 & 34 & 33 & 2 \\ 16 & 3 & 0 & 31 & 32 & 2 \\ 17 & 3 & 0 & 31 & 30 & 2 \\ 0 & 4 & 0 & 65 & 69 & 2 \\ & & & & & \end{array}$

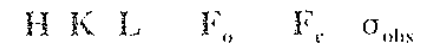

$\begin{array}{lllll}2 & 4 & 0 & 68 & 68 \\ 3 & 4 & 0 & 24 & 26\end{array}$

$440 \quad 138 \quad 135$

$640 \quad 44 \quad 42$

$740 \quad 73 \quad 72$

$8+0 \quad 29 \quad 30$

$10 \quad 4 \cdot 0 \quad 65 \quad 64$

$13+0+3+1$

$650+9 \quad 49$

$9 \begin{array}{llll}9 & 5 & 0 & 4\end{array}$

$1050 \quad 40 \quad 37$

$1350 \quad 36 \quad 35$

$\begin{array}{lllll}0 & 6 & 0 & 26 & 30\end{array}$

$\begin{array}{llllll}2 & 6 & 0 & 34 & 35\end{array}$

56

$\begin{array}{llll}7 & 6 & 0 & 29\end{array}$

$\begin{array}{rrrr}-20 & 0 & 1 & 70 \\ -18 & 0 & 1 & 100\end{array}$

$\begin{array}{rrrr}-18 & 0 & 100 \\ -16 & 0 & 170\end{array}$

$\begin{array}{lllll}-16 & 0 & 1 & 170 & 172\end{array}$

$\begin{array}{lllll}-14 & 0 & 1 & 45 & 45\end{array}$

$\begin{array}{lllll}-12 & 0 & 35 & 34\end{array}$

$\begin{array}{lllll}-8 & 0 & 1 & 132 & 127\end{array}$

$\begin{array}{lllll}-6 & 0 & 139 & 89\end{array}$

$\begin{array}{lllll}-4 & 0 & 1 & 648 & 622 \\ -2 & 0 & 1 & 129 & 134\end{array}$

$\begin{array}{lllll}0 & 0 & 105 & 106\end{array}$

$\begin{array}{lllll}2 & 0 & 1 & 608 & 590 \\ 4 & 0 & 1 & 244 & 245\end{array}$

$\begin{array}{lllll}6 & 0 & 4 & 43 & 40\end{array}$

$\begin{array}{lllll}8 & 0 & 1 & 75 & 72\end{array}$

$\begin{array}{lllll}10 & 0 & 132 & 131\end{array}$

$\begin{array}{lllll}12 & 0 & 18 & 21\end{array}$

$\begin{array}{lllll}14 & 0 & 1 & 133 & 131\end{array}$

$\begin{array}{rrrrr}16 & 0 & 1 & 66 & 63 \\ -19 & 1 & 1 & 21 & 20\end{array}$

$\begin{array}{lllll}-19 & 1 & 1 & 21 & 20 \\ -18 & 1 & 1 & 42 & 42\end{array}$

$\begin{array}{lllll}-17 & 1 & 74 & 74\end{array}$

$\begin{array}{llll}-15 & 1 & 45 & 47\end{array}$

$\begin{array}{llll}-14 & 1 & 73 & 74\end{array}$

$\begin{array}{llll}-13 & 1 & 1 & 45 \\ -12 & 1 & 1 & 24\end{array}$

$\begin{array}{lllll}-12 & 1 & 24 & 22 \\ -11 & 1 & 26 & 27\end{array}$

$\begin{array}{lllll}-10 & 1 & 84 & 81\end{array}$

$\begin{array}{llll}-9 & 1 & 36 & 34\end{array}$

$\begin{array}{lllll}-8 & 17+171\end{array}$

\begin{tabular}{l|lll}
-7 & 1 & 168 & 165
\end{tabular}

$\begin{array}{lllll}-6 & 1 & 53 & 54\end{array}$

$\begin{array}{lllll}-5 & 1 & 1 & 396 & 371\end{array}$

$-411623597$

\begin{tabular}{l|lll}
-3 & 1 & 843818
\end{tabular}

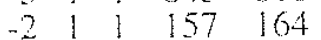

\begin{tabular}{l|lll}
-1 & 1 & 428 & 421
\end{tabular}

0113436

$\begin{array}{llll}1 & 1 & 412 & 390\end{array}$

21433411

$311 \quad 164 \quad 166$

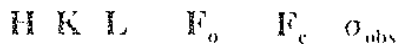

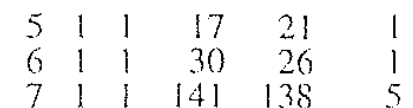

$\begin{array}{lllll}9 & 108 & 110 & 4\end{array}$

$\begin{array}{llllll}10 & 1 & 47 & 46 & 2\end{array}$

1) $1 \quad 117 \quad 115 \quad 4$

$141154 \quad 58$

$1811 \quad 27 \quad 26$

$\begin{array}{rrrrr}-18 & 2 & 1 & 48 & 48\end{array}$

$\begin{array}{rrrrrr}-16 & 2 & 1 & 23 & 23 & 2 \\ -14 & 2 & 1 & 53 & 54 & 2\end{array}$

$\begin{array}{llllll}-13 & 2 & 1 & 84 & 86 & 3\end{array}$

$\begin{array}{llllll}-12 & 2 & 1 & 14 & 15 & 2\end{array}$

$\begin{array}{llllll}-10 & 2 & 120 & 119 & 4\end{array}$

$\begin{array}{llllll}-9 & 2 & 30 & 31\end{array}$

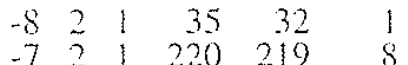

$\begin{array}{llllll}-6 & 2 & 1 & 357 & 350 & 12\end{array}$

\begin{tabular}{ll|lll}
-5 & 2 & 84 & 84 & 3
\end{tabular}

\begin{tabular}{ll|lll}
-42 & 163 & 156 & 6
\end{tabular}

$\begin{array}{rrrrrr}-3 & 2 & 1 & 17 & 15 & 2 \\ -2 & 2 & 194 & 185 & 7\end{array}$

$\begin{array}{llllll}-1 & 2 & 1 & 27 & 32\end{array}$

$\begin{array}{llllll}0 & 2 & 1 & 174 & 172 & 4\end{array}$

$\begin{array}{llllll}1 & 2 & 1 & 78 & 76 & 3\end{array}$

$\begin{array}{llllll}2 & 2 & 1 & 25 & 27 & 1 \\ 3 & 2 & 1 & 55 & 52 & 2\end{array}$

$\begin{array}{llllll}4 & 2 & 1 & 75 & 74 & 3\end{array}$

$\begin{array}{llllll}6 & 2 & 75 & 81 & 3\end{array}$

$\begin{array}{llllll}7 & 2 & 29 & 31 & 1\end{array}$

$\begin{array}{rrrrrr}8 & 2 & 1 & 96 & 101 & 3 \\ 9 & 2 & 1 & 89 & 93 & 3\end{array}$

$\begin{array}{llllll}10 & 2 & 1 & 109 & 112 & 4\end{array}$

$\begin{array}{llllll}11 & 2 & 45 & 45 & 2\end{array}$

12219195

$\begin{array}{llllll}14 & 2 & 1 & 30 & 29 & 2\end{array}$

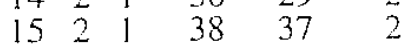

$\begin{array}{rrrrrr}16 & 2 & 1 & 24 & 28 & 2 \\ -17 & 3 & 1 & 46 & 46 & 2\end{array}$

$\begin{array}{lllll}-16 & 3 & 26 & 24\end{array}$

\begin{tabular}{rr|lll}
-15 & 3 & 34 & 38 & 2
\end{tabular}

$\begin{array}{lllll}-13 & 3 & 1 & 42 & 43\end{array}$

\begin{tabular}{rr|r}
-12 & 3138 & 36
\end{tabular}

$\begin{array}{lllll}-11 & 3 & 1 & 67 & 68\end{array}$

$\begin{array}{lllll}-9 & 3 & 1 & 83 & 82\end{array}$

\begin{tabular}{ll|ll}
-8 & 3 & 30 & 29
\end{tabular}

$\begin{array}{lllll}-7 & 3 & 124 & 122\end{array}$

\begin{tabular}{ll|ll}
-5 & 3 & 19 & 17
\end{tabular}

\begin{tabular}{rr|rr}
-3 & 3 & 1 & 80
\end{tabular}

\begin{tabular}{ll|ll}
-2 & 3 & 46 & 46
\end{tabular}

$\begin{array}{ccccc}-1 & 3 & 95 & 95\end{array}$

$\begin{array}{llllll}0 & 3 & 1 & 164 & 170 & 4\end{array}$

$\begin{array}{lllll}3 & 59 & 58 & 2\end{array}$

$3312424 \quad$

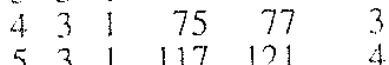

\begin{tabular}{rr|rrr}
5 & 3 & 177 & 121 & 4
\end{tabular} 


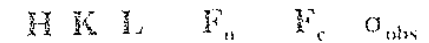

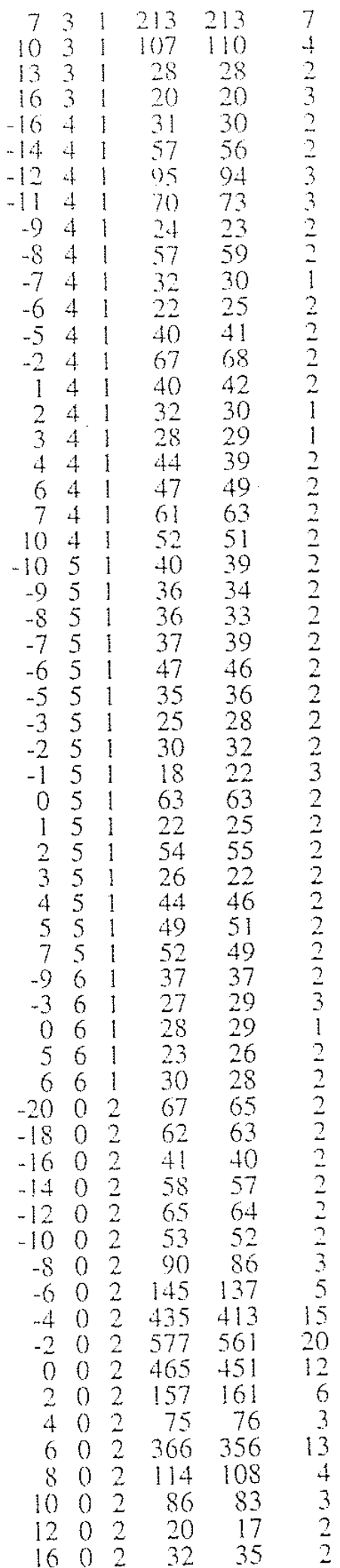

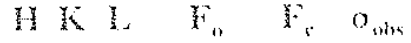

$\begin{array}{rrrrrr}18 & 0 & 2 & 26 & 24 & 3 \\ -20 & 1 & 2 & 30 & 32 & 2 \\ -19 & 1 & 2 & 23 & 24 & 2 \\ -18 & 1 & 2 & 20 & 23 & 3 \\ -17 & 1 & 2 & 65 & 66 & 2 \\ -15 & 1 & 2 & 20 & 23 & 2 \\ -14 & 1 & 2 & 43 & 41 & 2 \\ -13 & 1 & 2 & 53 & 53 & 2 \\ -12 & 1 & 2 & 86 & 86 & 3 \\ -11 & 1 & 2 & 78 & 76 & 3 \\ -8 & 1 & 2 & 76 & 77 & 3 \\ -7 & 1 & 2 & 358 & 350 & 13 \\ -6 & 1 & 2 & 95 & 89 & 3 \\ -5 & 1 & 2 & 73 & 70 & 3 \\ -4 & 1 & 2 & 142 & 139 & 5 \\ -3 & 1 & 2 & 105 & 109 & 4 \\ -2 & 1 & 2 & 396 & 383 & 14\end{array}$

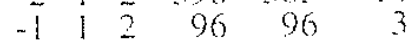

$\begin{array}{rrrrrr}0 & 1 & 2 & 72 & 71 & 2 \\ 1 & 1 & 2 & 189 & 183 & 7\end{array}$

$\begin{array}{llllll}2 & 1 & 2 & 46 & 42 & 2 \\ 3 & 1 & 2 & 44 & 42 & 2\end{array}$

$4 \quad 2 \quad 180 \quad 173$
5

$\begin{array}{lllll}5 & 1 & 2 & 41 & 40\end{array}$

$\begin{array}{lllll}6 & 2 & 44 & 44\end{array}$

$\begin{array}{lllll}7 & 1 & 2 & 64 & 62 \\ 8 & 1 & 2 & 54 & 53\end{array}$

$91287 \quad 87$

$\begin{array}{lllll}10 & 2 & 36 & 38\end{array}$

$11] 2 \quad 60 \quad 59$

$121239 \quad 38$

$\begin{array}{llll}15 & 1 & 2 & 43\end{array} 42$

$\begin{array}{lllll}18 & 1 & 2 & 27 & 26\end{array}$

$\begin{array}{rrrrr}-18 & 2 & 2 & 20 & 16 \\ -17 & 2 & 2 & 29 & 30\end{array}$

$\begin{array}{lllll}-17 & 2 & 2 & 29 & 30 \\ -15 & 2 & 2 & 23 & 24\end{array}$

$\begin{array}{lllll}-14 & 2 & 2 & 24 & 24\end{array}$

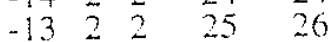

$\begin{array}{lllll}-13 & 2 & 2 & 25 & 56 \\ -12 & 2 & 2 & 57 & 56\end{array}$

$\begin{array}{rrrrr}-11 & 2 & 2 & 91 & 94 \\ -10 & 2 & 2 & 105 & 103\end{array}$

$\begin{array}{lll}-9 & 2 & 2\end{array}$

$\begin{array}{lll}-8 & 2 & 2\end{array}$

$\begin{array}{lll}-7 & 2 & 2 \\ -6 & 2 & 2\end{array}$

$\begin{array}{lllll}-6 & 2 & 2 & 102 & 101\end{array}$

$\begin{array}{lllll}-4 & 2 & 2 & 325 & 317\end{array}$

$\begin{array}{lllll}-3 & 2 & 2 & 60 & 51\end{array}$

$\begin{array}{lllll}-2 & 2 & 2 & 32 & 33\end{array}$

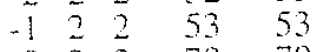

$\begin{array}{lllll}0 & 2 & 2 & 78 & 79\end{array}$

$\begin{array}{lllll}1 & 2 & 2 & 48 & 47\end{array}$

$2 \frac{2}{2} \quad \begin{array}{lll}2 & 130 & 129\end{array}$

$\begin{array}{llllll}4 & 2 & 2 & 124 & 115 & 4\end{array}$

$\begin{array}{llllll}5 & 2 & 2 & 84 & 84 & 3\end{array}$

$\begin{array}{rrrrrr}6 & 2 & 2 & 26 & 27 & 1 \\ 7 & 2 & 2 & 105 & 104 & 4\end{array}$

$\begin{array}{lllll}8 & 2 & 2 & 45 & 44\end{array}$

$\begin{array}{rrrrr}9 & 2 & 2 & 44 & 45 \\ 10 & 2 & 2 & 34 & 36\end{array}$

$\begin{array}{lllll}10 & 2 & 2 & 34 & 36 \\ 11 & 2 & 2 & 64 & 68\end{array}$

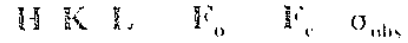

$\begin{array}{lllll}12 & 2 & 2 & 37 & 38 \\ 14 & 2 & 2 & 50 & 50 \\ 16 & 2 & 2 & 31 & 30\end{array}$

$\begin{array}{lllll}16 & 2 & 2 & 31 & 30 \\ 17 & 2 & 2 & 38 & 37\end{array}$

$\begin{array}{rrrrr}-16 & 3 & 2 & 48 & 46\end{array}$

$\begin{array}{rrrrr}-14 & 3 & 2 & 51 & 52 \\ -12 & 3 & 2 & 17 & 17\end{array}$

$\begin{array}{lllll}-11 & 3 & 2 & 161 & 163\end{array}$

$\begin{array}{lllll}-10 & 3 & 2 & 151 & 153\end{array}$

$\begin{array}{lllll}-9 & 3 & 2 & 148 & 151\end{array}$

$\begin{array}{lllll}-8 & 3 & 2 & 23 & 24\end{array}$

$\begin{array}{lllll}-7 & 3 & 2 & 52 & 49\end{array}$

$\begin{array}{lllll}-6 & 3 & 2 & 69 & 69 \\ -5 & 3 & 2 & 32 & 33\end{array}$

$\begin{array}{lllll}-3 & 3 & 2 & 56 & 55\end{array}$

$\begin{array}{lllll}-1 & 3 & 2 & 46 & 47\end{array}$

() $\begin{array}{llll}3 & 2 & 29 & 27\end{array}$

$\begin{array}{rrrr}3 & 2 & 18 & 5\end{array}$

$\begin{array}{llllll}2 & 3 & 2 & 59 & 59 & 2\end{array}$

$\begin{array}{llll}3 & 3 \cdot 2 & 53 & 5 \\ 5 & 3 & 83 & 8\end{array}$

$\begin{array}{lllll}6 & 3 & 2 & 88 & 9\end{array}$

$\begin{array}{lllll}7 & 3 & 2 & 17 & 18\end{array}$

$\begin{array}{lllll}8 & 3 & 2 & 22 & 24\end{array}$

$\begin{array}{rrrrr}9 & 3 & 2 & 64 & 65 \\ 11 & 3 & 2 & 49 & 48\end{array}$

$\begin{array}{lllll}12 & 3 & 2 & 23 & 24\end{array}$

$\begin{array}{lllll}14 & 3 & 2 & 40 & 40\end{array}$

$-1342 \quad 22 \quad 20$

$\begin{array}{rrrrr}-11 & 4 & 2 & 30 & 29 \\ -9 & 4 & 2 & 17 & 25\end{array}$

$\begin{array}{lllll}-8 & 4 & 2 & 33 & 35\end{array}$

$\begin{array}{llll}-4 & 4 & 2 & 32\end{array}$

$\begin{array}{rrrrr}-1 & 4 & 2 & 64 & 69\end{array}$

$\begin{array}{lllll}2 & 4 & 2 & 168 & 172\end{array}$

$342 \quad 110 \quad 113$

$\begin{array}{lllll}4 & 4 & 2 & 98 & 97 \\ 6 & 4 & 2 & 71 & 72\end{array}$

$\begin{array}{lllll}7 & 4 & 2 & 44 & 46\end{array}$

$945276 \quad 76$

1042

1142

$14 \quad 4222$

$\begin{array}{rlll}-11 & 52 & 34\end{array}$

$-852 \cdot 23$

$\begin{array}{lllll}-7 & 5 & 2 & 24 & 26\end{array}$

$\begin{array}{lllll}-6 & 5 & .2 & 21 & 17\end{array}$

$\begin{array}{lll}-4 & 5.2 & 33\end{array}$

$-35 ; 2$

$-1 \quad 5 \cdot 2$

$\begin{array}{llll}0 & 2 & -36 & 35\end{array}$

52
15443

$\begin{array}{lllll}3 & 5 & 2 & 58 & 61\end{array}$

$\begin{array}{lllll}6 & 5 & 2 & 35 & 32\end{array}$

$\begin{array}{lllll}-5 & 6 & 2 & 23 & 23\end{array}$

$\begin{array}{lllll}-3 & 6 & 2 & 26 & 32\end{array}$

$\begin{array}{llllll}-2 & 6 & 2 & 27 & 30 & 3\end{array}$

$\begin{array}{lllll}0 & 6 & 2 & 45 & 46\end{array}$

$\begin{array}{lllll}5 & 6 & 2 & 22 & 26 \\ 6 & 6 & 2 & 36 & 36\end{array}$

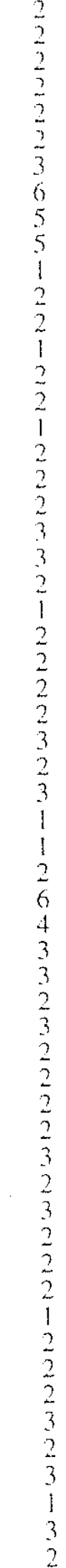




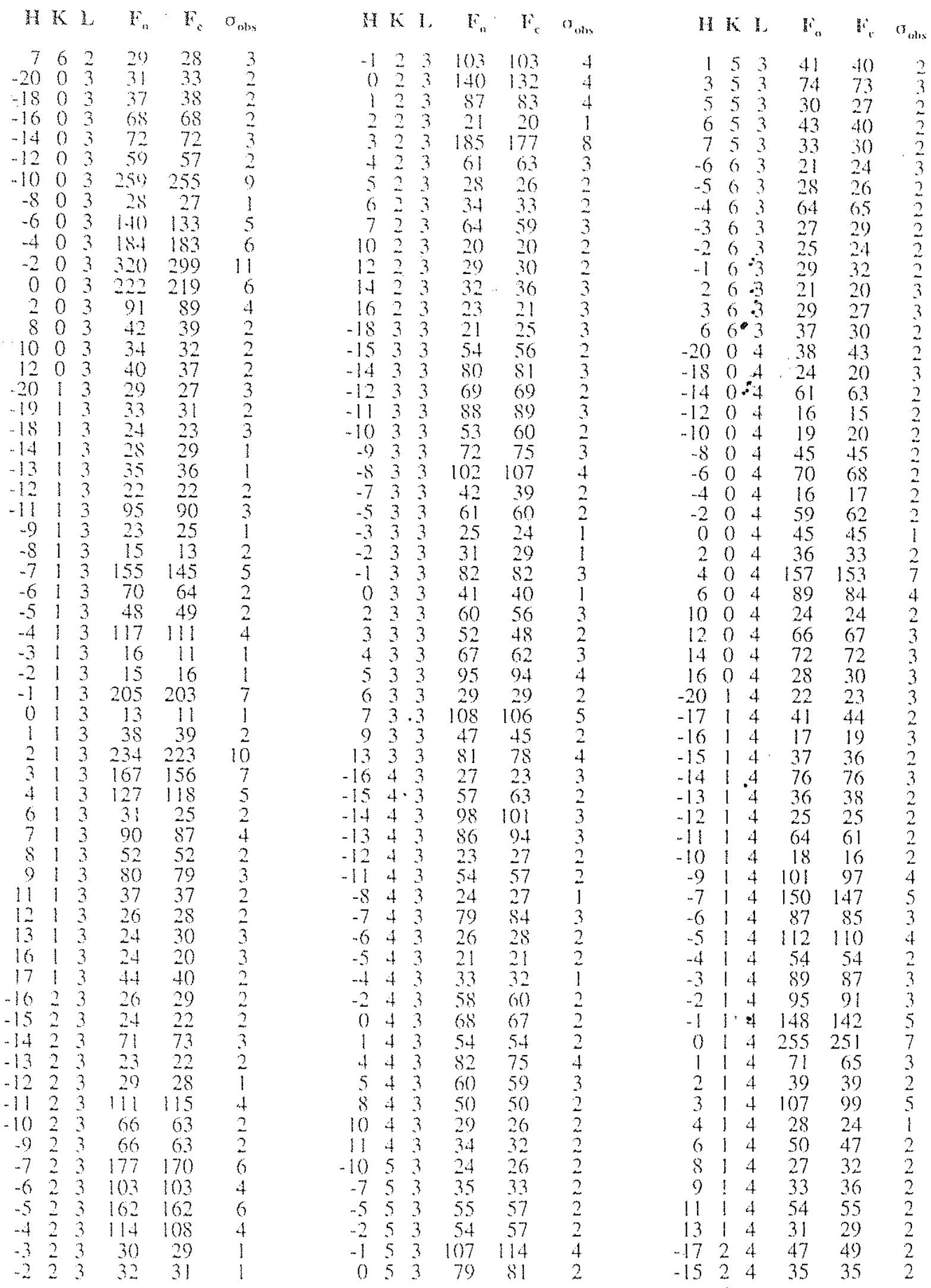




\begin{tabular}{|c|c|c|c|c|}
\hline $\mathrm{H}$ & $\mathrm{Kl}$ & & $F_{0}$ & $F_{c}$ \\
\hline 13 & 2 & it & 45 & 46 \\
\hline 12 & & 4 & 25 & 25 \\
\hline 11 & & 4 & 20 & 21 \\
\hline-6 & & 4 & & 39 \\
\hline 5 & & 4 & 72 & 74 \\
\hline-4 & & 4 & 4 & 41 \\
\hline 3 & & 4 & 19 & 20 \\
\hline & & 4 & 88 & \\
\hline 1 & & 4 & 175 & 173 \\
\hline & & 4 & 29 & 26 \\
\hline & 2 & 4 & 18 & 18 \\
\hline & & 4 & 47 & 49 \\
\hline & 2. & 4 & 138 & 129 \\
\hline & & 4 & 28 & 22 \\
\hline 6 & 2 & 4 & 62 & 62 \\
\hline 7 & 2 & 4 & 36 & 34 \\
\hline & $\tilde{2}$ & 4 & 25 & 26 \\
\hline & 2 & 4 & 79 & 82 \\
\hline 11 & 2 & 4 & 36 & 36 \\
\hline is & 2 & 4 & 30 & 26 \\
\hline 14 & 2 & 4 & 29 & 33 \\
\hline is & 3 & 4 & 36 & 38 \\
\hline 63 & 3 & 4 & 25 & 25 \\
\hline 14 & 3 & 4 & 71 & 75 \\
\hline 3 & 3 & 4 & 22 & 23 \\
\hline 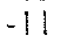 & 3 & 4 & 119 & 123 \\
\hline 10 & 3 & 4 & 27 & 25 \\
\hline 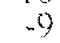 & 3 & 4 & 66 & 69 \\
\hline-7 & 3 & 4 & 64 & 65 \\
\hline-6 & 3 & 4 & 47 & 47 \\
\hline-5 & 3 & 4 & 112 & 114 \\
\hline & 3 & 4 & 33 & 34 \\
\hline-3 & 3 & 4 & 141 & 141 \\
\hline-2 & 3 & 4 & 34 & 33 \\
\hline 0 & 3 & 4 & 86 & 86 \\
\hline 1 & 3 & 4 & 20 & 18 \\
\hline 2 & 3 & 4 & 40 & 39 \\
\hline 3 & 3 & 4 & 39 & 37 \\
\hline & 2 & 4 & 33 & 31 \\
\hline & 3 & 4 & 37 & 39 \\
\hline & 3 & 4 & 49 & 52 \\
\hline 11 & 3 & 4 & 56 & 57 \\
\hline & 3 & 4 & 32 & 32 \\
\hline-1 & 54 & 4 & 57 & 57 \\
\hline-14 & 44 & 4 & 22 & 20 \\
\hline & 24 & 4 & 56 & 56 \\
\hline-11 & 1 & 4 & 69 & 7.3 \\
\hline 10 & it & 4 & 29 & 29 \\
\hline (1) & 4 & 4 & 30 & 3.3 \\
\hline , & it & 4 & 34 & 35 \\
\hline & 4 & 4 & 55 & 56 \\
\hline & it & 4 & 67 & 69 \\
\hline & 4 & 4 & 20 & 20 \\
\hline & 4 & 4 & 18 & 13 \\
\hline & 4 & 4 & 6.5 & 61 \\
\hline & 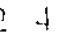 & 4 & 35 & 32 \\
\hline & 1 & 4 & 65 & 61 \\
\hline & & 4 & 41 & 39 \\
\hline & & 4 & 46 & 46 \\
\hline & & & 24 & 25 \\
\hline
\end{tabular}

HKL F F

$\begin{array}{rrrrr}9 & + & 66 & 62 & 3 \\ 10 & +4 & 27 & 24 & 2\end{array}$

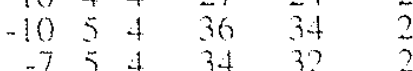

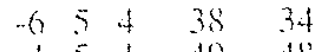

$-45+4948$

$-1542220$

$05+2018$

1545954

$\begin{array}{rrrr}2 & 54 & 41 & 67 \\ 3 & 5 & 113 & 105\end{array}$

$+544943$

$55+4038$

$75+7368$

$\begin{array}{lllll}-9 & 6 & 4 & 55 & 48\end{array}$

$\begin{array}{lllll}-8 & 6 & 4 & 26 & 26 \\ -5 & 6 & 4 & 61 & 62\end{array}$

$-46+49+49$

$-26+2424$

$\begin{array}{lllll}-1 & 6 & 4 & 49 & 49\end{array}$

$\begin{array}{rrrrr}-14 & 0 & 5 & 48 & 48\end{array}$

$\begin{array}{lllll}-12 & 0 & 5 & 66 & 63 \\ -10 & 0 & 5 & 17 & 15\end{array}$

$\begin{array}{rrrrr}-10 & 0 & 5 & 17 & 15 \\ -8 & 0 & 5 & 53 & 55\end{array}$

$\begin{array}{lllll}-6 & 0 & 5 & 27 & 26\end{array}$

$\begin{array}{lllll}-4 & 0 & 5 & 456 & 418\end{array}$

$\begin{array}{rrrrr}-2 & 0 & 5 & 23 & 20 \\ 0 & 0 & 5 & 97 & 90\end{array}$

$\begin{array}{lllll}2 & 0 & 5 & 117 & 116\end{array}$

$\begin{array}{lllll}4 & 0 & 5 & 102 & 108\end{array}$

$\begin{array}{lllll}6 & 0 & 5 & 132 & 132\end{array}$

$\begin{array}{lllll}8 & 0 & 5 & 31 & 3.1\end{array}$

$1000551 \quad 52$

$\begin{array}{lllll}-18 & 1 & 5 & 56 & 56\end{array}$

$\begin{array}{lllll}-17 & 5 & 53 & 22\end{array}$

$\begin{array}{lllll}-16 & 1 & 5 & 39 & 40\end{array}$

$-13 \quad 5 \quad 27$

$\begin{array}{llll}-12 & 1 & 5 & 30\end{array}$

\begin{tabular}{l|ll}
-11 & 5 & 17
\end{tabular}

$-10 \quad 1546$

\begin{tabular}{l|lll}
-8 & 5 & 4 & 4 \\
-7 & 1 & 5 & 125
\end{tabular}

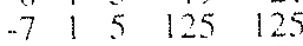

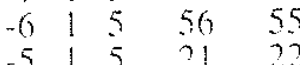

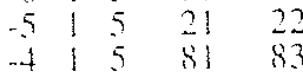

$-3 \quad 5 \quad 4848$

$\begin{array}{lllll}2 & 5 & 54 & 0.4\end{array}$

$\begin{array}{lllll}-1 & 5 & 84 & 77\end{array}$

$\begin{array}{lllll}0 & 1 & 5 & 65 & 62\end{array}$

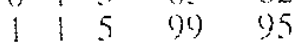

$\begin{array}{llll}2 & 5 & 52 & 52 \\ 4 & 5 & 41 & 42\end{array}$

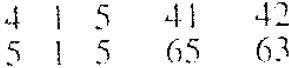

\begin{tabular}{l|lll}
6 & 5 & 45 & 50
\end{tabular}

\begin{tabular}{l|lll}
9 & 5 & 89 & 92
\end{tabular}

\begin{tabular}{l|lll}
11 & 1 & 5 & 30
\end{tabular}

$13 \quad 5 \quad 46 \quad 48$

$\begin{array}{rrrrr}14 & 1 & 5 & 37 & 36 \\ -17 & 2 & 5 & 41 & 42\end{array}$

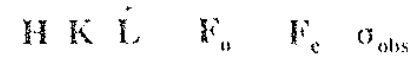

$-15 \div 5 \quad 58 \quad 60 \quad 2$

$\begin{array}{lllll}-14 & 2 & 5 & 33 & 35\end{array}$

$\begin{array}{lllll}-12 & 2 & 5 & 48 & 48\end{array}$

$\begin{array}{lllll}-11 & 2 & 5 & 16 & 19 \\ -10 & 2 & 5 & 12 & 45\end{array}$

$\begin{array}{rrrrr}-9 & 2 & 5 & 102 & 99 \\ -8 & 2 & 5 & 31 & 33\end{array}$

$\begin{array}{lllll}-7 & 2 & 5 & 87 & 84\end{array}$

$\begin{array}{llll}-6 & 2.5 & 23 & 24\end{array}$

$\begin{array}{lllll}-5 & 2 & 5 & 59 & 6.3\end{array}$

$\begin{array}{lllll}-4 & 2 & 5 & 28 & 32 \\ -3 & 2 & 5 & 89 & 0.4\end{array}$

$\begin{array}{lllll}-2 & 2 & 5 & 59 & 57\end{array}$

$\begin{array}{llll}0 & 5 & 122 & 117\end{array}$

2 2.5.5. $55 \quad 55$

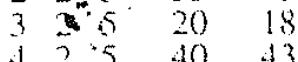

$\begin{array}{lllll}6 & 2 & 5 & 79 & 85\end{array}$

$\begin{array}{lllll}9 & 2 & 5 & 58 & 57\end{array}$

$\begin{array}{lllll}10 & 2 & 5 & 115 & 116\end{array}$

$\begin{array}{lllll}11 & 2 & 5 & 46 & 47 \\ 14 & 2 & 5 & 27 & 30\end{array}$

$\begin{array}{lllll}-18 & 3 & 5 & 45 & 41\end{array}$

$\begin{array}{lllll}-15 & 3 & 5 & 30 & 28\end{array}$

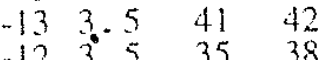

$-113 \cdot 5 \quad 15 \quad 18$

$\begin{array}{lllll}-9 & 3 & 5 & 70 & 75\end{array}$

$\begin{array}{lllll}-8 & 3 & 5 & 30 & 31\end{array}$

$\begin{array}{ccccc}-6 & 3 & 5 & 20 & 24 \\ -5 & 3 & 5 & 51 & 50\end{array}$

$\begin{array}{lllll}-5 & 3 & 5 & 51 & 50 \\ -4 & 3 & 5 & 89 & 91\end{array}$

$\begin{array}{rrrrr}-4 & 3 & 5 & 89 & 91 \\ -3 & 3 & 5 & 99 & 102\end{array}$

$\begin{array}{lllll}-2 & 3 & 5 & 18 & 16\end{array}$

$\begin{array}{lllll}0 & 3 & 5 & 86 & 88\end{array}$

$\begin{array}{lllll}3 & 3 & 56 & 42\end{array}$

$\begin{array}{lllll}3 & 3 & 5 & 47 & 46\end{array}$

$\begin{array}{lllll}4 & 3 & 5 & 33 & 33\end{array}$

$\begin{array}{lllll}5 & 3 & 5 & 36 & 38\end{array}$

$\begin{array}{lllll}8 & 3 & 5 & 33 & 31\end{array}$

$\begin{array}{lllll}9 & 3 & 5 & 28 & 27\end{array}$

1135545

$\begin{array}{llll}12 & 3 & 5 & 45\end{array}$

$\begin{array}{lllll}13 & 3 & .5 & 78 & 77\end{array}$

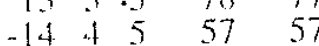

$\begin{array}{rrrrr}-10 & 4 & 5 & 47 & 48\end{array}$

$\begin{array}{llll}-9 & 4 & 5 & 38 \\ -8 & 30\end{array}$

$\begin{array}{lllll}-8 & 4 & 5 & 33 & 35\end{array}$

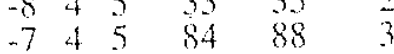

$\begin{array}{lllll}-5 & +5 & 29 & 29 & 1\end{array}$

$\begin{array}{lllll}-3 & 4 & 5 & 26 & 27\end{array}$

$\begin{array}{rrrrrr}-2 & 4 & 5 & 48 & 47 & 2 \\ -1 & 4 & 5 & 113 & 117 & 4\end{array}$

$\begin{array}{lllll}0 & 4 & 5 & 147 & 147\end{array}$

$\begin{array}{lllll}1 & 4 & 5 & 46 & 45\end{array}$

$\begin{array}{llllll}2 & 4 & 5 & 22 & 20 & 2\end{array}$

$\begin{array}{llllll}3 & 4 & 5 & 81 & 81 & 4 \\ 7 & 4 & 5 & 33 & 35 & 2\end{array}$

1) $4 \begin{array}{llll}4 & 23 & 20 & 3\end{array}$ 


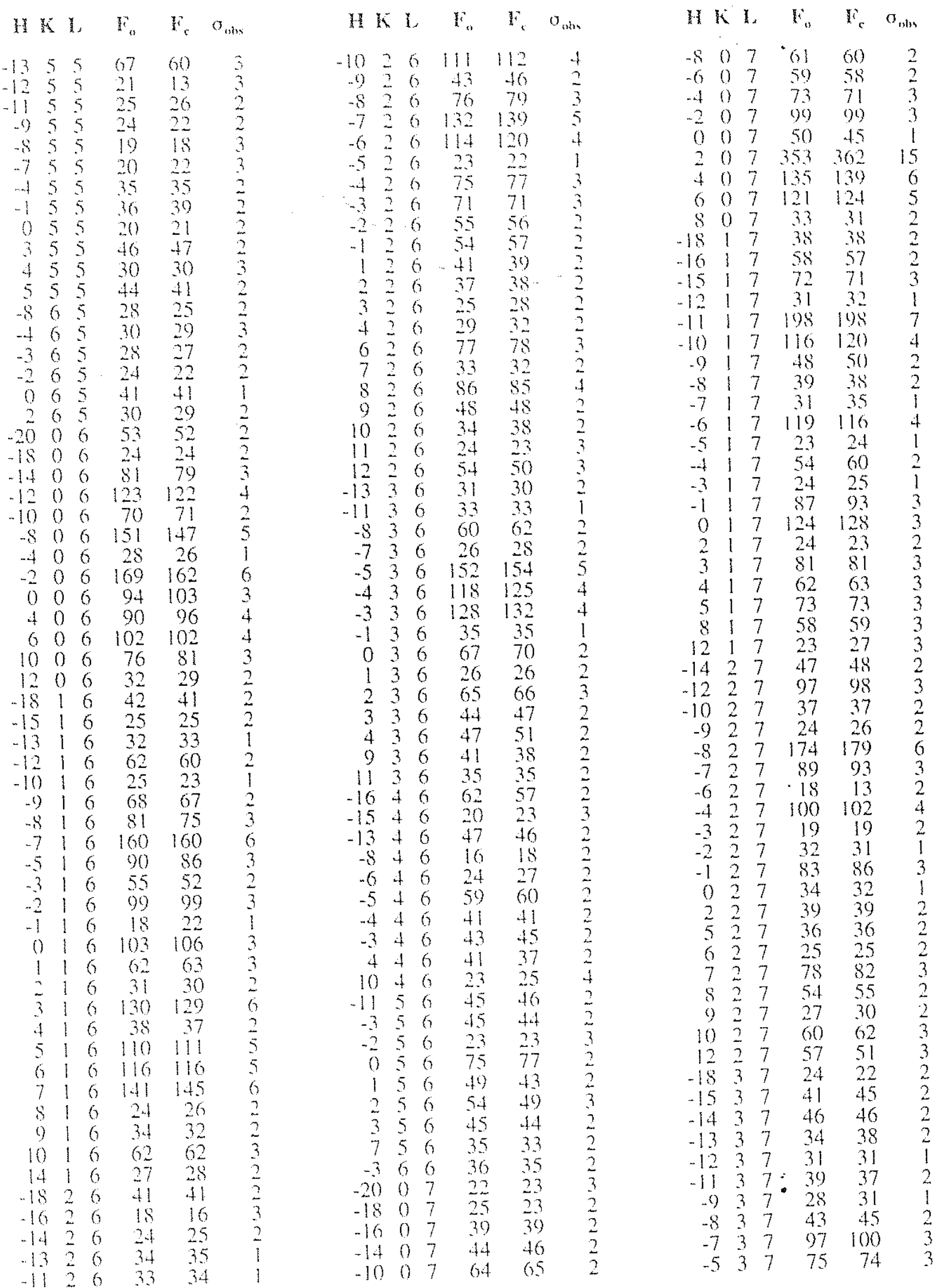




\begin{tabular}{|c|c|c|c|c|c|c|c|c|c|c|c|c|c|c|c|c|c|}
\hline $7 x$ & $\mathrm{~K}$ & l. & $F_{0}$ & 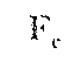 & $\sigma_{0, x}$ & $H$ & $\mathrm{~K}$ & 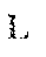 & $E_{n}$ & $l^{1}$ & $\sigma_{\text {ond }}$ & $H$ & $K$ & 1. & $w_{11}$ & $\because{ }^{\prime}$ & $\mathrm{O}_{\mathrm{ob}}$ \\
\hline-4 & 3 & 7 & 17 & 16 & 2 & -2 & 4 & 7 & 92 & 95 & 3 & 6 & 0 & 8 & 28 & 26 & 2 \\
\hline-3 & 3 & 7 & 6.3 & 63 & 2 & $\overline{0}$ & 4 & 7 & 69 & 70 & 2 & 8 & 0 & 8 & 38 & 30) & 2 \\
\hline 2 & 3 & 7 & 45 & 49 & 2 & 2 & 4 & 7 & +7 & 45 & 2 & 10 & 0 & 8 & 62 & 66 & 3 \\
\hline-1 & 3 & 7 & 30 & 31 & 1 & 6 & 4 & 7 & 75 & 67 & 3 & -19 & 1 & 8 & 27 & 23 & 2 \\
\hline () & 3 & 7 & 16 & 19 & 2 & -11 & 5 & 7 & 33 & 26 & 2 & -17 & 1 & 8 & 54 & 51 & 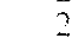 \\
\hline 2 & 3 & 7 & 36 & 37 & $\overline{2}$ & -0 & 5 & 7 & 20 & 19 & 3 & -16 & 1 & 8 & 53 & 53 & 2 \\
\hline 3 & 3 & 7 & 42 & 41 & 2 & -8 & 5 & 7 & .37 & 33 & 2 & -15 & 1 & 8 & 70 & 70 & 3 \\
\hline 4 & 3 & 7 & 40 & 49 & 2 & -7 & 5 & 7 & 33 & 30 & 2 & -14 & 1 & 8 & 29 & 29) & 1 \\
\hline 5 & 3 & 7 & 33 & 33 & 2 & -6 & 5 & 7 & 28 & 26 & 2 & -12 & 1 & $S$ & 54 & $\$ 5$ & 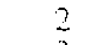 \\
\hline 6 & 3 & 7 & 26 & 24 & 2 & -4 & 5 & 7 & 49 & 48 & 2 & -11 & 1 & 8 & 76 & 77 & 0 \\
\hline 8 & 3 & 7 & 31 & 29 & 2 & -2 & 5 & 7 & 32 & 30 & 2 & -9 & 1 & 8 & 33 & 31 & $\frac{1}{3}$ \\
\hline 9) & 3 & 7 & 27 & 29 & 2 & -18 & 0 & 8 & 70 & 72 & 3 & -8 & 1 & 8 & 79 & 78 & 3 \\
\hline 10 & 3 & 7 & 51 & 50 & 2 & -16 & 0 & 8 & 71 & 72 & 3 & .6 & 1 & 8 & 54 & 57 & \\
\hline-11 & 4 & 7 & 19 & 21 & 3 & -14 & 0 & 8 & 237 & 236 & 8 & -5 & 1 & 8 & 46 & 44 & \\
\hline-8 & 4 & 7 & 37 & 37 & 2 & -12 & 0 & 8 & 19 & 17 & 2 & -4 & 1 & 8 & 60 & 6.3 & \\
\hline-7 & 4 & 7 & 33 & 33 & 2 & -10 & 0 & 8 & 90 & 95 & 3 & -3 & 1 & 8 & 44 & 47 & \\
\hline-6 & 4 & 7 & 29 & 28 & 2 & -6 & 0 & 8 & 25 & 28 & 2 & -2 & 1 & 8 & 125 & 1.3 .4 & 4 \\
\hline-5 & 4 & 7 & 37 & 38 & 2 & -2 & 0 & 8 & 116 & 134 & 4 & -1 & 1 & 8 & 175 & 185 & \\
\hline-4 & 4 & 7 & 31 & 36 & 2 & 0 & 0 & 8 & 29 & 32 & 1 & 0 & 1 & 8 & 62 & (2) & \\
\hline-3 & 4 & 7 & 62 & 63 & 2 & 4 & 0 & 8 & 120 & 124 & 5 & 1 & 1 & 8 & 33 & 34 & \\
\hline 2 & 1 & 8 & 20 & 22 & 2 & 3 & 3 & 8 & 27 & 24 & 2 & -17 & 1 & 9 & 69 & 67 & \\
\hline 3 & $i$ & $S$ & 110 & 114 & 5 & 4 & 3 & 8 & 26 & 25 & 2 & -15 & 1 & 9 & 82 & 80 & \\
\hline 4 & $i$ & 8 & 89 & 90 & 4 & 6 & 3 & 8 & 54 & 55 & 3 & -13 & 1 & 9 & 60 & 60 & \\
\hline 5 & 1 & 8 & 40 & 42 & 2 & 7 & 3 & 8 & 30 & 23 & 2 & -12 & 1 & 9 & 49 & 51 & \\
\hline 6 & 1 & 8 & 65 & 66 & 3 & 8 & 3 & 8 & 25 & 16 & 3 & -11 & 1 & 9 & 32 & 34 & \\
\hline 7 & 1 & 8 & 29 & 33 & 2 & 9 & 3 & 8 & 89 & 81 & 4 & -10 & 1 & 9 & 55 & 56 & \\
\hline 8 & $i$ & 8 & 29 & 33 & 2 & -14 & 4 & 8 & 34 & 30 & 2 & -7 & 1 & 9 & 20 & 19 & \\
\hline 9 & $i$ & 8 & 32 & 28 & 2 & -12 & 4 & 8 & 34 & 30 & 2 & -6 & 1 & 9 & 39 & 39 & \\
\hline-18 & 2 & 8 & 32 & 34 & 2 & .10 & 4 & 8 & 76 & 73 & 3 & -5 & 1 & 9 & 91. & 95 & \\
\hline-16 & 2 & 8 & 29 & 27 & 2 & -9 & 4 & 8 & 28 & 29 & 2 & -3 & 1 & 9 & $54^{\circ}$ & 60 & \\
\hline-14 & 2 & 8 & 52 & 51 & 2 & -8 & 4 & 8 & 23 & 21 & 3 & -2 & 1 & 9 & 32 & 34 & \\
\hline-12 & 2 & 8 & 43 & 44 & 2 & -7 & 4 & 8 & 62 & 61 & 2 & 0 & 1 & 9 & 112 & 115 & \\
\hline-11 & 2 & 8 & 95 & 06 & 3 & -6 & 4 & 8 & 4 & 42 & 2 & 1 & $i$ & 9 & $\cdot 26$ & 28 & \\
\hline-10 & 2 & 8 & 23 & 25 & 2 & -4 & 4 & 8 & 48 & 48 & 2 & 3 & 1 & 9 & 50 & 51 & \\
\hline-9 & 2 & 8 & 68 & 71 & 2 & -3 & 4 & 8 & 36 & 35 & 2 & 6 & 1 & 9 & 72 & 71 & \\
\hline-8 & 2 & 8 & 71 & 74 & 3 & -1 & 4 & 8 & 27 & 27 & 2 & 10 & 1 & 9 & 36 & 36 & \\
\hline-6 & 2 & 8 & 37 & 38 & 2 & 0 & 4 & 8 & 41 & 39 & 1 & -18 & 2, & $g$ & 33 & 33 & \\
\hline-.5 & 2 & 8 & 94 & 96 & 3 & 4 & 4 & 8 & 29 & 31 & 2 & -15 & 2 & 6) & 24 & 25 & \\
\hline-4 & 2 & 8 & 152 & 157 & 5 & -8 & 5 & 8 & 38 & 35 & 2 & -14 & 2 & 9 & 40 & 39 & \\
\hline-3 & 2 & 8 & 61 & 61 & 2 & -5 & 5 & 8 & 42 & 40 & 2 & -10 & 2 & 9 & 28 & 31 & \\
\hline 0 & 2 & 8 & 83 & 90 & 2 & -4 & 5 & 8 & 45 & 44 & 2 & -9 & 2 & $\Rightarrow$ & 51 & 49 & \\
\hline 2 & 2 & 8 & 50 & 52 & 2 & -1 & 5 & 8 & 44 & 45 & 2 & -8 & $i$ & 9 & 54 & 55 & \\
\hline 4 & 2 & 8 & 41 & 40 & 2 & 0 & 5 & 8 & 24 & 22 & 2 & -6 & 2 & 9 & 97 & 101 & \\
\hline 8 & 2 & 8 & 28 & 27 & 3 & 2 & 5 & 8 & 31 & 27 & 2 & -5 & 2 & 9 & 74 & 74 & \\
\hline 9 & 2 & 8 & 24 & 22 & 3 & -18 & 0 & 9 & 90 & 97 & 4 & -4 & 2 & 9 & 67 & 71 & \\
\hline 10 & 2 & 8 & 29 & 27 & 3 & -16 & 0 & ) & 20 & 19 & 2 & -2 & 2. & 9 & 117 & 121 & \\
\hline .18 & 3 & 8 & 28 & 27 & 2 & -12 & 0 & $?$ & 90 & 90 & 4 & 0 & 2 & 9 & 19 & 21 & \\
\hline-16 & 3 & 8 & 23 & 16 & 3 & -10 & 0 & 9 & 28 & 30 & 1 & 1 & 2 & () & 24 & 25 & \\
\hline-15 & 3 & 8 & 39 & 38 & 2 & -8 & 0 & 9 & 26 & 24 & 2 & 2 & 2 & 9 & 43 & 43 & \\
\hline-14 & 3 & 8 & 22 & 20 & 3 & -4 & 0 & 0 & 52 & 57 & 2 & 3 & 2 & 9 & 40 & 42 & \\
\hline-12 & 3 & 8 & 25 & 24 & 2 & -2 & 0 & 0 & 50 & 52 & 2 & 5 & 2 & 9 & 36 & 30 & \\
\hline-11 & 3 & 8 & 50 & 61 & 2 & 0 & 0 & () & 131 & 138 & 4 & 6 & 2 & 9 & 37 & 38 & \\
\hline .10 & 3 & 8 & 52 & 50 & 2 & 2 & 0 & 0 & 101 & 103 & 4 & 7 & 2 & 9 & 30 & 28 & \\
\hline-7 & 3 & 8 & 46 & 47 & 2 & 4 & 0 & ) & 30 & 32 & 2 & ) & 2 & 9 & 28 & 26 & \\
\hline-6 & 3 & 8 & 32 & 34 & 1 & 6 & 0 & 9 & 57 & 60 & 3 & -15 & 3 & 9 & 24 & 19 & \\
\hline-5 & 3 & 8 & 62 & 63 & 2 & 8 & 0 & 9 & 75 & 72 & 3 & -14 & 3 & 9 & 61 & 59 & \\
\hline-1 & 3 & 8 & 46 & 53 & 2 & 10 & 0 & ) & 30 & 31 & 2 & -12 & 3 & 9 & 23 & 20 & \\
\hline 0 & 3 & 8 & 20 & 20 & 2 & -10 & 1 & 9 & 69 & 68 & 3 & -10 & 3 & 9 & 35 & 38 & \\
\hline 1 & 3 & 8 & 22 & 20 & 2 & -18 & 1 & 9 & 47 & $4 ?$ & 2 & -9 & 3 & 9 & 95 & 95 & \\
\hline 2 & 3 & 8 & 82 & 80 & 4 & & & & & & & & & & & & \\
\hline
\end{tabular}




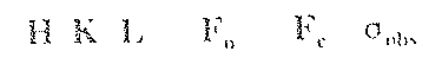

$\begin{array}{rrrrrr}-8 & 3 & 9 & 64 & 64 & 2 \\ -7 & 3 & 9 & 136 & 135 & 5 \\ -4 & 3 & 9 & 6.4 & 65 & 2\end{array}$

$\begin{array}{rrrrrr}-4 & 3 & 9 & 64 & 65 & 2 \\ -1 & 3 & 9 & 35 & 37 & 2\end{array}$

$\begin{array}{rrrr}439 & 32 & 32 \\ -11 & 4 & 30 & 30\end{array}$

$\begin{array}{rrrr}-4 & 49 & 23 & 24\end{array}$

$\begin{array}{lllll}1 & 4 & 9 & 28 & 25 \\ 3 & 4 & 9 & 32 & 32\end{array}$

$\begin{array}{rrrrr}3 & 4 & 9 & 32 & 32 \\ 4 & + & 9 & 67 & 54\end{array}$

$\begin{array}{lllll}5 & 49 & 36 & 29 & 2\end{array}$

$\begin{array}{llllll}-9 & 5 & 9 & 34 & 20 & 2 \\ -4 & 5 & 0 & 24 & 20 & 2\end{array}$

$\begin{array}{llllll}-4 & 5 & 9 & 24 & 20 & 2 \\ -2 & 5 & 9 & 33 & 33 & 2 \\ -1 & 5 & 9 & 40 & 40 & 2\end{array}$

$\begin{array}{llll}-18 & 010 & 57 & 55\end{array}$

-16010 44 44

$\begin{array}{rrrr}-14 & 010 & 46 & 47 \\ -12 & 010 & 30 & 33\end{array}$

$\begin{array}{rlll}-8 & 010 & 113 & 116\end{array}$

$\begin{array}{lllll}-6 & 010 & 24 & 22 \\ + & 010 & 71 & 79\end{array}$

$\begin{array}{llll}-2 & 010 & 51 & 50\end{array}$

$\begin{array}{llll}0 & 010 & 75 & 75\end{array}$

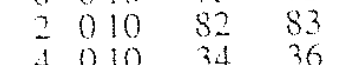

6010

$-18 \quad 110$

$-16 \quad 110$

$-13110$

$-10 \quad 110$

-9) 110

$-5110$

$-3110$

$-2110$

111061

$+11051$

511030

6110

8110

$-18210$

$-14210$

$-12 \quad 210$

$-11210$

.10210

$\begin{array}{ll}-9 & 210\end{array}$

$-8 \quad 210$

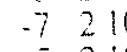

$\begin{array}{lll}-5 & 2 & 10\end{array}$

+210
$-3 \quad 210$

$-3 \quad 210$

$-2 \quad 210$

0
0 10

$\begin{array}{lll}2 & 2 & 10\end{array}$

$\begin{array}{lll}3 & 210\end{array}$

$+210$

5210

$\begin{array}{rr}7 & 210 \\ -13 & 310\end{array}$

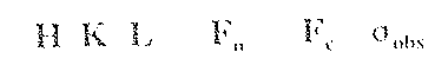

$\begin{array}{cccccc}-12 & 310 & 45 & 43 & 2 \\ -11 & 3 & 10 & 22 & 22 & 2 \\ -10 & 310 & 24 & 23 & 2 \\ -9 & 310 & 41 & 42 & 2\end{array}$

$\begin{array}{llll}-9 & 3 & 40 & 4 \\ -8 & 10 & 4\end{array}$

$\begin{array}{llll}-7 & 310 & 43 & 42\end{array}$

$\begin{array}{llll}-6 & 310 & 36 & 3 \\ -5 & 310 & 56 & 5\end{array}$

$-3.310$

() 310

$5 \quad 310$

$-12+10$

$-11+10$

$-10+10$

$-8+10$

$-6+10$

$-5410$

$-4410$

$-3+10$

() +10

2410

$\begin{array}{lll}-18 & 011\end{array}$

$-16011$

$-14011$

$-12011$

$-1001$

$\begin{array}{lll}-6 & 011\end{array}$

$-4011$

$\begin{array}{llll}6 & 0 & 11\end{array}$

$-15 \mid 11$

$-12 \quad 111$

$-10111$

$\begin{array}{lll}-9 & 11\end{array}$

$-7111$

$\begin{array}{lll}-5 & 111\end{array}$

$-4 \quad 11$

$\begin{array}{lll}-3 & 1 & 11 \\ -2 & 1 & 11\end{array}$

$-2111$

$\begin{array}{llll}0 & 1 & 11 \\ 1 & 1 & 1 & 1\end{array}$

211

$\begin{array}{lll}3 & 1 & 11 \\ 4 & 1 & 11\end{array}$

4
41
-1421
-41

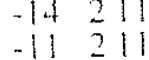

$-1021$

$\begin{array}{ll}-9 & 2\end{array}$

$-4211$

$\begin{array}{lll}-3 & 2 & 11\end{array}$

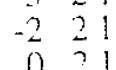

1211

2211

5211

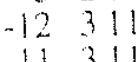

$-11311$

-) 311

$\begin{array}{lll}-7 & 311\end{array}$

$\begin{array}{ll}-5 & 31\end{array}$
$29 \quad 32 \quad 2$

$26 \quad 24 \quad \frac{2}{76} \quad 75$

$54 \quad 55$

$\begin{array}{ll}38 & 37 \\ 36 & 37\end{array}$

1) 20

535

28

21

34

50)

$+2$

41
20

40

23

$37 \quad 35$

$35 \quad 36$

$30 \quad 27$

$27 \quad 28$

5154

19 14

$54 \quad 53$

39

40
35

$4 !$
42

4) 32

$50 \quad 51$

24

20

27

19
52

$26 \quad 25$

28

23

34
28

38

29

$22 \quad 24$

$40 \quad 37$

$19 \quad 21$

$\begin{array}{ll}61 & 61 \\ 57 & 59\end{array}$

30

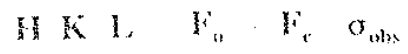

$\begin{array}{rrllll}-4 & 311 & 37 & 38 & 2 \\ -3 & 311 & 25 & 17 & 2\end{array}$

$-131106 \quad 66$

$\begin{array}{llll}2 & 311 & 52 & 48\end{array}$

$\begin{array}{rrrrr}-10 & 411 & 44 & 43 \\ 9 & 411 & 44 & 42\end{array}$

$\begin{array}{lll}43 & -12 & 2\end{array}$

$\begin{array}{lllll}-6 & 411 & 23 & 26 & 3 \\ -3 & 411 & 20 & 28 & 3\end{array}$

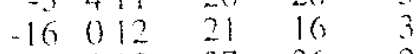

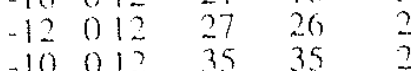

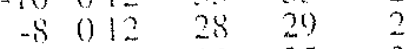

$\begin{array}{lllll}-4 & 012 & 33 & 35 & 2\end{array}$

$\begin{array}{lllll}-2 & 0 & 12 & 89 & 89\end{array}$

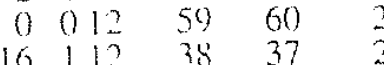

$\begin{array}{lllll}-15 & 112 & 26 & 23 & 2\end{array}$

$\begin{array}{lllll}-14 & 112 & 42 & 40 & 2\end{array}$

$\begin{array}{llllll}-13 & 1 & 12 & 31 & 29 & 2\end{array}$

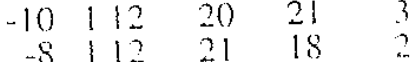

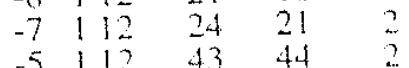

$\begin{array}{llllll}-3 & 112 & 33 & 34 & 2\end{array}$

$\begin{array}{rrrrrr}-2 & 1 & 12 & 20 & 16 & 3 \\ -15 & 2 & 12 & 33 & 30 & 2\end{array}$

$\begin{array}{lllll}-13 & 212 & 20 & 17 & 3\end{array}$

$\begin{array}{lllll}-11 & 212 & 37 & 32 & 2\end{array}$

$\begin{array}{llllll}-8 & 2 & 12 & 24 & 23 & 2 \\ -7 & 2 & 12 & 41 & 38 & 2\end{array}$

$\begin{array}{llllll}-5 & 2 & 12 & 45 & 46 & 2\end{array}$

$\begin{array}{lllll}-3 & 211 & 24 & 26 & 36\end{array}$

$\begin{array}{llllll}2 & 2 & 12 & 44 & 44 & 2\end{array}$

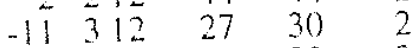

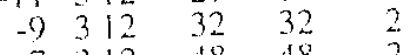

$\begin{array}{lllll}-7 & 3 & 12 & 48 & 4\end{array}$

$\begin{array}{llllll}-3 & 3 & 12 & 33 & 29\end{array}$

$\begin{array}{lllll}-2 & 3 & 12 & 29 & 25\end{array}$

$\begin{array}{lllll}-1 & 3 & 12 & 34 & 32\end{array}$

$\begin{array}{rrrrr}-12 & 0 & 13 & 37 & 32 \\ -10 & 0 & 13 & 54 & 53\end{array}$

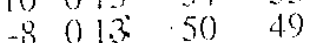

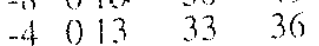

$\begin{array}{lllll}0 & 0 & 13 & 33 & 32\end{array}$

$\begin{array}{llll}-7 & 13 & 19 & 22\end{array}$

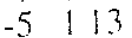

$-1113$

$0 \quad 113$

$\begin{array}{lll}-12 & 213\end{array}$

$\begin{array}{llll}-11 & 2 & 13\end{array}$

$-9213$

$-8213$

$-7 \quad 213$

$\begin{array}{ll}-5 & 2 \\ 3\end{array}$

$\begin{array}{lll}-4 & 213\end{array}$

$\begin{array}{llllll}-3 & 213 & 26 & 52 & 2\end{array}$

$\begin{array}{rrrrr}-10 & 0 & 14 & 21 & 17\end{array}$

$\begin{array}{llllll}-8 & 0 & 14 & 26 & 19 & 2 \\ -6 & 0 & 14 & 19 & 16 & 2\end{array}$ 


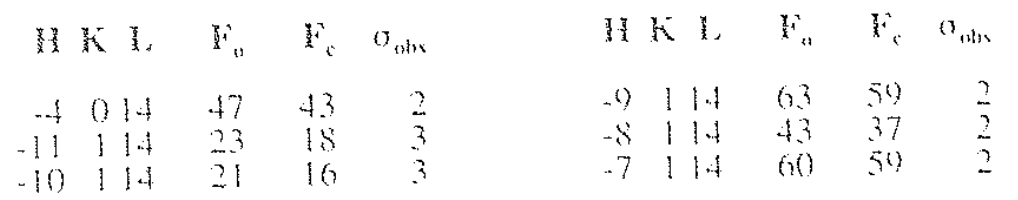

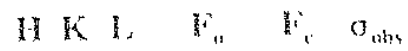
$\begin{array}{lllll}-4 & 14 & 30 & 30 & 2\end{array}$

$\therefore$ 